\title{
Genetical Relationships among Some Populations of a Lancelet, Branchiostoma belcheri, in the Western Japan
}

\author{
Koichiro Kawai $^{1)}$, Akira Ishikawa ${ }^{2)}$, Hidetoshi Saito ${ }^{1)}$ and Hiromichi Imabayashi ${ }^{1)}$ \\ 1) Laboratory of Aquatic Ecology, Graduate School of Biosphere Science, Hiroshima University, \\ Kagamiyama 1-4-4, Higashihiroshima-shi, Hiroshima, 739-8528 Japan, \\ ${ }^{2)}$ Welcome Graxo Co. Ltd., Ogori-cho 3-26-601, Yoshiki-gun, Yamaguchi, 754-0014 Japan
}

\begin{abstract}
Genetical differences were examined among three geographically different populations of a lancelet, Branchiostoma belcheri in western Japan. Electrophoresis of PCR products with six different primers detected 13-19 DNA bands from sixteen individuals of these three populations. Among them, two bands were common to all individuals, and three bands were shared with the populations of Hiroshima Bay and the Sea of Genkai. One band was specific to the population of the Sea of Genkai, and three bands were specific to the population of Osaka Bay. In cluster analysis on the genetic similarity, the individuals collected at the Sea of Genkai are relatively close to those of Hiroshima Bay, but both populations are relatively distant from those of Osaka Bay. It might be inferred that the three populations have not originated from a common larval recruitment and there are no genetical exchanges via larval recruitment at least between the populations of Hiroshima Bay and Osaka Bay.
\end{abstract}

Key words: Branchiostoma belcheri, genetical relationship, lancelet, population, RAPD

\section{INTRODUCTION}

Lancelets, called "Namekujiuo" in Japanese, are a member of the subphylum Cephalochordata of the phylum Chordata. They have been proved to be the closest invertebrate relative of the vertebrates based on the phylogenetic tree with DNA sequence (Wada and Satoh, 1994). They live in relatively coarse sand at the bottom of the sea and spend most of their time in the shallow burrows filter-feeding small particles, especially phytoplankton (Stokes and Holland, 1998). After leaving planktonic life in the form of amphioxus larva for two or three months after hatching, they settle on the bottom, metamorphose and live benthic life (Wickstead, 1975).

Received June 13, 2003: Accepted November 12, 2003
In Japan, three lancelet species, Branchiostoma belcheri Gray, Asymmetron maldivense (ForsterCooper) and A. lucayanum Andrews, are distributed. Among these, $B$. belcheri is widely distributed in the Pacific coastal region of middle to western Japan (Nishikawa, 1995). Many samples of planktonic larvae have been collected at the points along the Kuroshio Current (Shojima, 1974). However, it is still not clear whether the adult populations distributed along the coastal areas of the Japanese Archipelagoes are sustained by recruits of planktonic larvae via the Kuroshio Current. Unfortunately, the lancelet populations have declined year to year, especially in the Seto Inland Sea and the adjacent areas (Nishikawa, 1998). To clarify the mechanisms by which the lancelet populations in the Seto Inland Sea are sustained, studies should be conducted on populations in this region from a genetical viewpoint.

In this study, we collected sixteen individuals of lancelet at three different places in western Japan, the 
Sea of Genkai, Hiroshima Bay and Osaka Bay, and analysed the genetic relationship and diversity among these three populations, using randomly amplified polymorphic DNA (RAPD) technique, which has been widely used to analyse genetic polymorphism of fishes (Foo, Dinesh, Lim, Chan \& Phang, 1995; Allegrucci, Caccone, Cataudella, Powell and Sbordoni, 1995; Miyanohara, Iwatsuki and Sakai, 1999).

\section{MATERIALS AND METHODS}

\section{Survey areas}

We collected the lancelets at three different places in the Seto Inland Sea and the adjacent seas (Hiroshima Bay, the Sea of Genkai and Osaka Bay in western Japan)

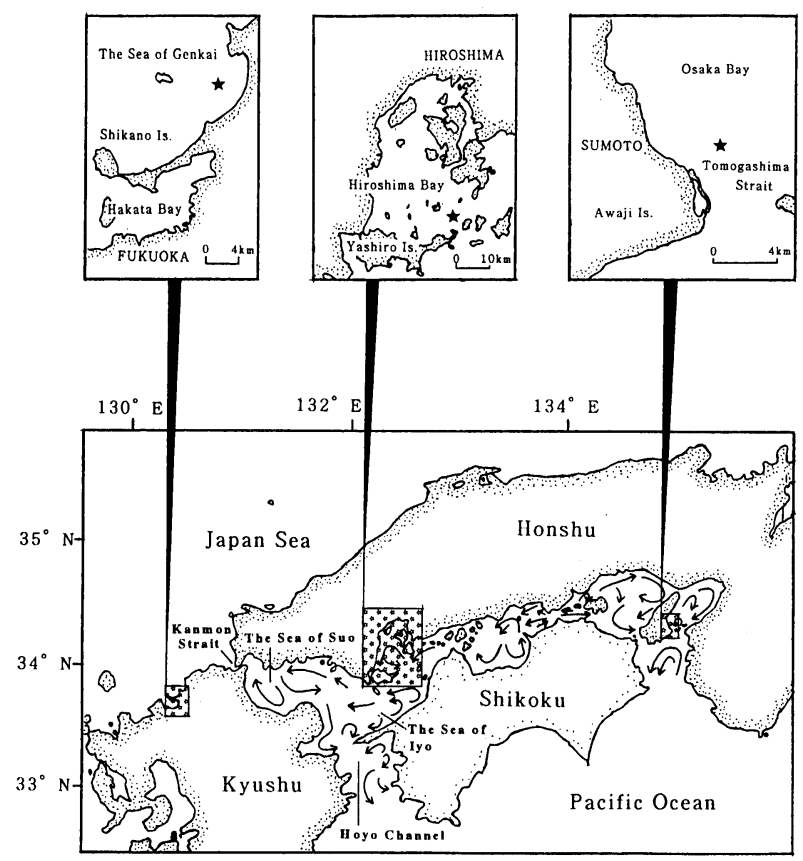

Fig. 1. Map of the Western Japan, showing the locations of 3 sampling sites. Arrows indicate the direction of water current according to Yanagi and Higuchi (1979) and Itami (1984).
(Fig. 1) in 1997 and 1998 (Table 1). Depth at the collection site was 50-60, 10 and $60 \mathrm{~m}$, for Hiroshima Bay, the Sea of Genkai and Osaka Bay, respectively. For particle size composition in the sediment, clay $(<63 \mu \mathrm{m})$ and conglomerate $(2 \mathrm{~mm}<)$ were higher than 10 and 20 $\%$ in volume, respectively, for Hiroshima Bay whereas they were nearly $0 \%$ and lower than $6 \%$, respectively, for the other two seas. In contrast, sand $(63 \mu \mathrm{m}-2 \mathrm{~mm})$ was lower than $70 \%$ for Hiroshima Bay whereas it was higher than $90 \%$ for the other two seas (Table 1).

\section{Lancelet sampling}

We performed lancelet sampling on the Trainee of Hiroshima University, called Toyoshiomaru (320 displacement tonnage), using a Smith-McIntire type grab sampler $\left(0.1 \mathrm{~m}^{2}\right.$ in sampling area). Collected sediment samples were sieved with a sieve of $1 \mathrm{~mm}$ in mesh. Lancelets were collected from the residential materials on the mesh with a forceps. We transported lancelet samples to the laboratory in alive conditions, and then stored them at $-80{ }^{\circ} \mathrm{C}$ before analysis.

\section{RAPD analysis}

We prepared template DNA for PCR using an EasyDNA $^{\mathrm{TM}}$ Kit (Invitrogen, San Diego, CA, USA), according to the manufacturer's instruction. Prior to RAPD analysis, the whole body of frozen lancelets was sliced into chips.

We performed RAPD analysis using a kit (ReadyTo-Go RAPD Analysis Beads, Pharmacia, Uppsala, Sweden) with a thermal cycler (THERMO PROCESSOR TR-100, Taitec, Koshigaya, Saitama, Japan). A set of 6 different oligonucleotides (RAPD Analysis Primer Set, Pharmacia, Uppsala, Sweden) was used as PCR primers. The sequences were: 5'-GGTGCGGGAA-3' for Primer 01, 5'-GTTTCGCTCC-3' for Primer 02, 5'GTAGACCCGT-3' for Primer 03, 5'-AAGAGCCCGT-3' for Primer 04, 5'-AACGCGCAAC-3' for Primer 05 and 5'-CCCGTCAGCA-3' for Primer 06. Two different DNA

Table 1. Particle size composition of the sediment and lancelet samples. Numbers in parentheses indicate mean body length.

\begin{tabular}{|c|c|c|c|c|c|c|}
\hline \multirow{2}{*}{$\begin{array}{c}\text { Survey } \\
\text { area }\end{array}$} & \multirow[t]{2}{*}{ Period } & \multicolumn{3}{|c|}{ Particle size composition $(\%)$} & \multicolumn{2}{|c|}{ Lancelet samples } \\
\hline & & mud & sand & gravel & No. samples & Body length $(\mathrm{mm})$ \\
\hline Hiroshima Bay & Apr. 28, '97-Jul. 7, ' 98 & 11 & 67 & 22 & 8 & $\begin{array}{c}20.0-27.4 \\
(23.8)\end{array}$ \\
\hline Sea of Genkai & Sep. 24, ' 97 & 0 & 95 & 5 & 3 & $\begin{array}{c}40.5-49.7 \\
\quad(44.0)\end{array}$ \\
\hline Osaka Bay & Feb. $18,{ }^{\prime} 98$ & 0 & 94 & 6 & 5 & $\begin{array}{c}27.1-35.3 \\
\quad(31.5)\end{array}$ \\
\hline
\end{tabular}


polymerase, AmpliTaq ${ }^{\mathrm{TM}}$ polymerase and Stoffel fragment, were used. Prepared DNA (450 ng) was used as a template. PCR was performed in $25 \mu \mathrm{L}$ of a buffer containing $3 \mathrm{mM} \mathrm{MgCl}_{2}, 30 \mathrm{mM} \mathrm{KCl}$ and $10 \mathrm{mM}$ Tris, pH8.3, using the following cycle profile: one cycle at 95 ${ }^{\circ} \mathrm{C}$ for 5 minutes followed by 45 cycles at $95{ }^{\circ} \mathrm{C}$ for 1 minute, $36{ }^{\circ} \mathrm{C}$ for 1 minute and $72{ }^{\circ} \mathrm{C}$ for 2 minutes, and by one cycle at $72{ }^{\circ} \mathrm{C}$ for 7 minutes. After electrophoresing in an agarose gel plate $(1.2 \%)$ at $150 \mathrm{~V}$, the PCR products were stained with ethidium bromide and detected under ultraviolet illumination.

\section{Dissimilarity (D) value}

We calculated D value (Gilbert et al., 1990) as an indicator of the genetical distance among the different populations in the following formula:

$\mathrm{D}=\mathrm{N}_{a b} /\left(\mathrm{N}_{a}+\mathrm{N}_{b}\right)$

where $\mathrm{N}_{a b}$ was the number of fragments which were not shared by individuals a and $\mathrm{b} ; \mathrm{N}_{a}$ and $\mathrm{N}_{b}$ were the number of fragments for individuals $a$ and $b$, respectively.

We calculated the mean $\mathrm{D}$ value of the results using six primers for each combination of individuals and performed a cluster analysis on the basis of the mean value. The results were expressed with a dendrogram by the UPG (Unweighted Pair-Group Clustering) method (Nei, 1975).

\section{RESULTS}

We collected sixteen individuals of lancelet in total (Table 1). The mean body length was smallest in Hiroshima Bay $(23.8 \mathrm{~mm})$, and largest in the Sea of Genkai $(44.0 \mathrm{~mm})$.

\section{RAPD analysis with six different primers}

[Electrophoresis of PCR products with Primer 01]

We detected fourteen DNA bands from all of the collected individuals, which were numbered in order from the electrophoretic origin (Fig. 2). There were specific bands to a population and common bands to multiple populations. Band 12 was specific to the individuals of Osaka Bay. Band 5 was common to all the individuals of Hiroshima Bay and the Sea of Genkai. Bands 4 and 6 were common to all the individuals of the Sea of Genkai and also detected in the Hiroshima Bay population. Band 7 was common to all the individuals of the Sea of Genkai and also detected in the other two populations. Band 13 was common to all the individuals of Osaka Bay and also detected in the Hiroshima Bay population. Band 9 was common to all the individuals of Osaka Bay and also detected in the other two populations. Band 11 was detected in all the populations.

[Electrophoresis of PCR products with Primer 02] We detected sixteen DNA bands with Primer 02 (Fig. $3)$. Band 6 was common to all the individuals of $\mathrm{Hi}$ roshima Bay and also detected in the population of the Sea of Genkai. Bands 11 and 14 were common to all the individuals of the Sea of Genkai and also detected in the other two populations. Bands 7 and 12 were common to all the individuals of Osaka Bay and also detected in the other two populations. Bands 8 and 15 were detected in all the populations. Bands 2 and 10 were detected only in the Sea of Genkai and the Hiroshima Bay populations, respectively.

[Electrophoresis of PCR products with Primer 03] We detected nineteen DNA bands with Primer 03 (Fig. 4). Bands 10 and 17 were specific to the individuals of the Sea of Genkai and Osaka Bay, respectively. Band 14 was common to all the individuals of all the populations. Band 13 was common to all the individuals of the Sea of Genkai and also detected in the Hiroshima Bay population. Band 16 was detected in all the populations. Bands 11,12 and 19 were detected only in the Hiroshima Bay, the Sea of Genkai and the Osaka Bay populations, respectively.

[Electrophoresis of PCR products with Primer 04] We detected eighteen DNA bands with Primer 04 (Fig. 5). Bands 13 and 14 were common to all the individuals of the Sea of Genkai and Osaka Bay and also detected in the Hiroshima Bay population. Band 12 was common to all the individuals of the Sea of Genkai and also detected in the other two populations. Bands 7, 10, 11 and 16 were detected in all the populations. Bands 9 and 15 were detected only in the Sea of Genkai and Osaka Bay populations, respectively.

[Electrophoresis of PCR products with Primer 05] We detected sixteen DNA bands with Primer 05 (Fig. 6). Bands 6 and 8 were each specific to the individuals of the Sea of Genkai and Osaka Bay, respectively. Band 7 was common to all the individuals of Hiroshima Bay and the Sea of Genkai. Band 12 was common to all the individuals of Hiroshima Bay and also detected in the other two populations. Band 9 was common to all the individuals of the Sea of Genkai and also detected in the 
other two populations. Band 5 was common to all the individuals of Osaka Bay and also detected in the other two populations. Band 11 was detected in all the populations. Bands 1, 2 and 13; and band 16 were detected only in the Sea of Genkai and in the Osaka Bay populations, respectively.

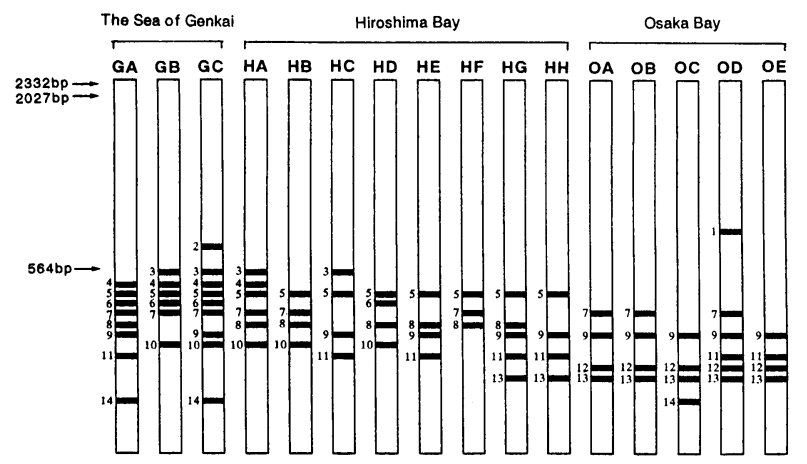

Fig. 2. Electropholetic pattern of PCR products using a Primer 01. Each lane shows the datum for each individual. Arrows at the left show the DNA size markers.

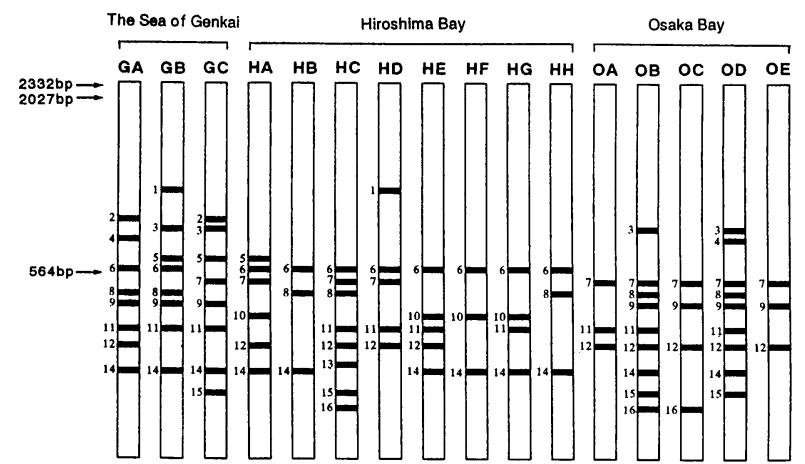

Fig. 3. Electropholetic pattern of PCR products using a Primer 02 .

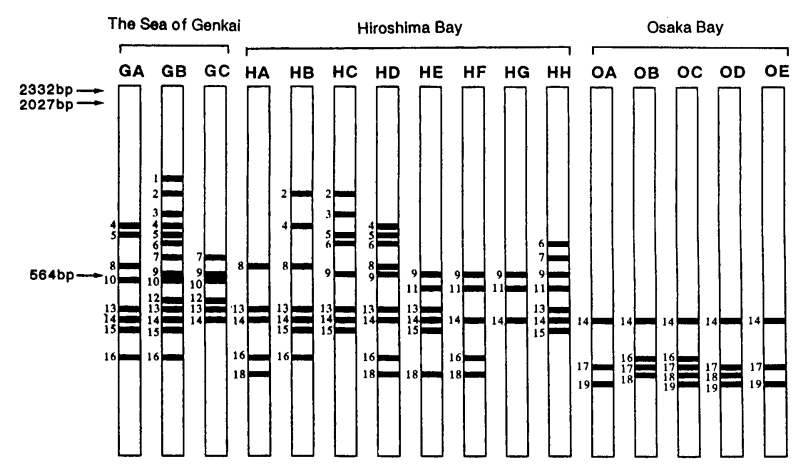

Fig. 4. Electropholetic pattern of PCR products using a Primer 03.
[Electrophoresis of PCR products with Primer 06]

We detected thirteen DNA bands with Primer 06 (Fig. 7). Band 10 was common to all the individuals of all the populations. Band 9 was common to all the individuals of Hiroshima Bay and the Sea of Genkai. Band 6 was common to all the individuals of the Sea of Genkai and also detected in the Hiroshima Bay population. Band 12

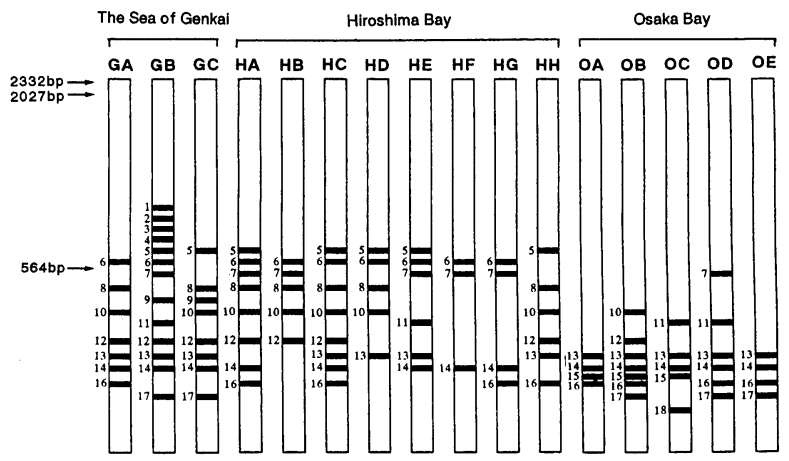

Fig. 5. Electropholetic pattern of PCR products using a Primer 04.

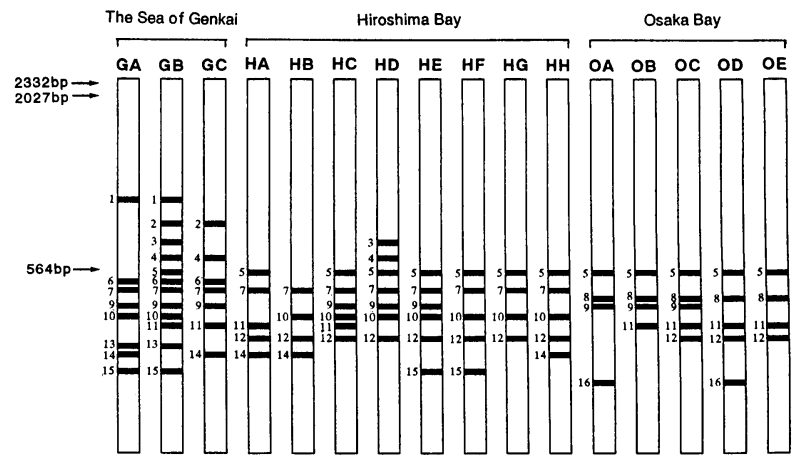

Fig. 6. Electropholetic pattern of PCR products using a Primer 05 .

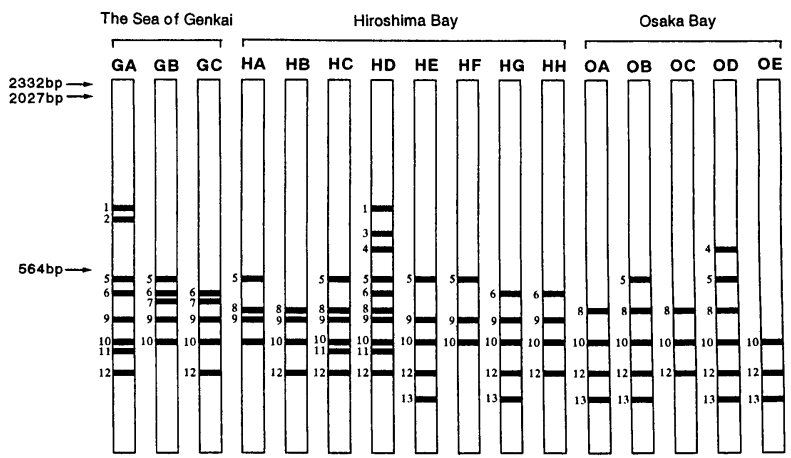

Fig. 7. Electropholetic pattern of PCR products using a Primer 06. 
was common to all the individuals of Osaka Bay and also detected in the other two populations. Band 5 was detected in all the populations. Band 7 was detected only in the Sea of Genkai population.

\section{$D$ values and cluster analysis}

We calculated the mean $\mathrm{D}$ values using six primers with the results of electrophoresis for each combination of individuals. A matrix of $\mathrm{D}$ values between the individuals was shown in Table 2 . The $\mathrm{D}$ values between the individuals of Hiroshima Bay were in the range of 0.23-0.58 and those between the individuals of the Sea of Genkai were in the range of $0.33-0.43$, whereas those between the individuals of Osaka Bay were in the range of 0.230.34 . The $\mathrm{D}$ values between the individuals of Hiroshima Bay and the Sea of Genkai (0.40-0.70) were lower than those between the individuals of Hiroshima Bay and Osaka Bay (0.48-0.83) and those between the individuals of the Sea of Genkai and Osaka Bay (0.56-0.81).

Two large clusters were constructed: the Hiroshima Bay-Sea of Genkai cluster and the Osaka Bay cluster (Fig. 8). All the individuals of Hiroshima Bay make a single cluster at a D value within 0.46 . Those of Osaka Bay make a single cluster by themselves at a $D$ value of about 0.29. An individual from the Sea of Genkai (GA) constructed a small cluster with an individual from Hiroshima Bay. The other two individuals of the Sea of Genkai (GB and GC), making a small cluster by themselves, participated in the cluster of Hiroshima Bay. The Hiroshima Bay and the Osaka Bay populations became a single cluster at a D value higher than 0.6.

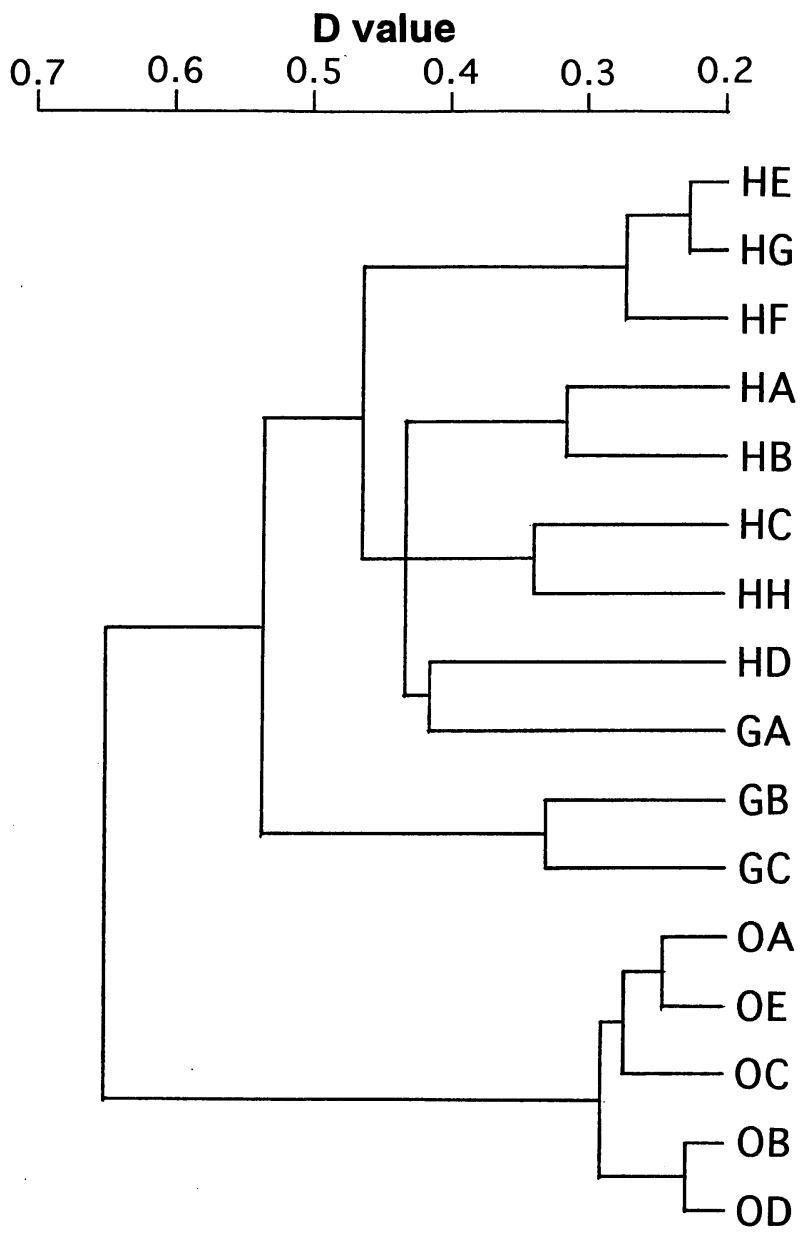

Fig. 8. A dendrogram of sixteen lancelet samples from three sea areas on the basis of $\mathrm{D}$ values.

Table 2. A matrix of $D$ values among a total of sixteen individuals from three sea areas. The mean of values using six different primers is shown.

\begin{tabular}{|c|c|c|c|c|c|c|c|c|c|c|c|c|c|c|c|}
\hline & GA & GB & GC & HA & HB & $\mathrm{HC}$ & HD & HE & HF & HG & HH & OA & OB & OC & OD \\
\hline GB & 0.409 & & & & & & & & & & & & & & \\
\hline GC & 0.425 & 0.329 & & & & & & & & & & & & & \\
\hline HA & 0.463 & 0.488 & 0.495 & & & & & & & & & & & & \\
\hline HB & 0.415 & 0.543 & 0.580 & 0.317 & & & & & & & & & & & \\
\hline HC & 0.404 & 0.487 & 0.496 & 0.410 & 0.473 & & & & & & & & & & \\
\hline HD & 0.415 & 0.472 & 0.542 & 0.409 & 0.443 & 0.377 & & & & & & & & & \\
\hline HE & 0.440 & 0.543 & 0.591 & 0.424 & 0.485 & 0.348 & 0.420 & & & & & & & & \\
\hline HF & 0.563 & 0.547 & 0.695 & 0.344 & 0.401 & 0.578 & 0.538 & 0.271 & & & & & & & \\
\hline HG & 0.563 & 0.626 & 0.631 & 0.501 & 0.487 & 0.465 & 0.550 & 0.226 & 0.275 & & & & & & \\
\hline HH & 0.443 & 0.558 & 0.493 & 0.502 & 0.356 & 0.339 & 0.506 & 0.376 & 0.529 & 0.327 & & & & & \\
\hline $\mathbf{O A}$ & 0.679 & 0.799 & 0.691 & 0.675 & 0.800 & 0.587 & 0.678 & 0.594 & 0.793 & 0.587 & 0.696 & & & & \\
\hline OB & 0.567 & 0.636 & 0.559 & 0.515 & 0.676 & 0.481 & 0.647 & 0.569 & 0.665 & 0.625 & 0.617 & 0.261 & & & \\
\hline OC & 0.696 & 0.781 & 0.673 & 0.613 & 0.765 & 0.566 & 0.661 & 0.596 & 0.736 & 0.690 & 0.697 & 0.290 & 0.268 & & \\
\hline OD & 0.635 & 0.670 & 0.645 & 0.560 & 0.714 & 0.534 & 0.687 & 0.502 & 0.647 & 0.545 & 0.625 & 0.296 & 0.228 & 0.340 & \\
\hline OE & 0.702 & 0.808 & 0.680 & 0.580 & 0.830 & 0.568 & 0.740 & 0.580 & 0.781 & 0.542 & 0.606 & 0.246 & 0.325 & 0.255 & 0.248 \\
\hline
\end{tabular}




\section{DISCUSSION}

All the individuals of Osaka Bay make a cluster by themselves at a $\mathrm{D}$ value of about 0.29 whereas those of $\mathrm{Hi}$ roshima Bay make a cluster at about 0.46 . Further, the genetical dissimilarity of Osaka Bay population was much lower ( 0.34 at the highest $D$ value) than that of Hiroshima Bay (0.58 at the highest). This suggests that the gene pool size of the former population be much smaller than the latter. The reason for this is unknown. Examination of many more samples from more populations in the Osaka Bay area and detailed investigation of various environmental factors, possibly causing reduction or patchiness of habitats, are necessary to elucidate the mechanisms for this phenomenon.

Among all the DNA bands, bands 5, 7 and 9 were common to all the individuals of Hiroshima Bay and the Sea of Genkai using Primers 01, 05 and 06, respectively, and these were not detected in any individual of Osaka Bay. In contrast, bands 12,17 and 8 were common to all the individuals of Osaka Bay using Primers 01, 03 and 05 , respectively, and these were not detected in any individual of the other two populations. This also suggests the (mutual) genetical exchange or common origin between the Hiroshima Bay and the Sea of Genkai populations. However, further detailed examination, using quite different designs of PCR primer, should be performed before conclusion.

Why is the Osaka Bay population genetically distant from the Hiroshima Bay population? A possible explanation is as follows. Each of the open seas (Nada) in the Seto Inland Sea is considered to be oceanographically highly isolated (Endo et al., 1984). In addition, exchanging movement of sea water in the Seto Inland Sea is practically interrupted by the existence of a sea ridge, dividing this sea into the eastern and western portions, in the Bisan Seto which is situated between Hiroshima and Osaka Bays (Itami, 1984). Thus, there might be a barrier to mutual exchange of planktonic lancelet larvae.

Then, why is the population of the Sea of Genkai genetically near to that of Hiroshima Bay? It could be explained by a relatively high possibility of mutual (or one-way) recruitment of planktonic larvae between these populations. That is, there is a large scale of counterclockwise water movement in the Seas of Suo and Iyo (Yanagi and Higuchi, 1979; Itami, 1984), both adjacent to Hiroshima Bay, caused by a strong inflow of oceanic water through the Hoyo Channel which has by far the largest minimum sectional area among all the channels opening from the Seto Inland Sea to the Pacific Ocean
(Higuchi, 1976). Further, there is a high velocity of water current through the Kanmon Strait (Yanagi and Higuchi, 1979), lying as a barrier between the two populations. Such conditions, together with a long planktonic period (Wickstead, 1975), might make the abovementioned mutual recruitment possible. However, more detailed studies should be conducted on many more populations from as many areas scattered on the whole Seto Inland Sea as possible before conclusion.

In this study, an individual of the Sea of Genkai was affiliated to a cluster by the Hiroshima Bay population. This might suggest the possibility of a common recruitment source for these populations. The source is possibly the population brought by Kuroshio Current. However, this possibility should be carefully examined by further genetical analyses of the samples from the multiple sites along the Current.

The life cycle of lancelet, including sea areas for oviposition, routes and duration of migration at planktonic stages and movement during development after settlement, has not been clarified for populations in the Seto Inland Sea and the adjacent areas. Further studies are essential to elucidate the causation of the genetical relationships between the populations.

\section{REFERENCES}

Allegrucci, G., Caccone, A., Cataudella, S., Powell, J.R. and Sbordoni, V. 1995. Acclimation of the European sea bass to freshwater: monitoring genetic changes by RAPD polymerase chain reaction to detect DNA polymorphism. Marine Biology, 121: 591-599.

Endo, T., H. Takeoka and H. Tsubota 1984. Water movement and quality in the Seto Inland Sea. In, General Study for Environmental Assessment on the Seto Inland Sea. Reports of Co-operative Research (Preservation of Environment) Ministry of Education, Science and Culture, pp. 49-97 (in Japanese)

Foo, C.L., Dinesh, K.R., Lim, T.M., Chan, W.K. and Phang, V.P.E. 1995. Inheritance of RAPD bands in the guppy fish, Poecilia reticulata. Zoological Science, 12: 535-541.

Gilbert, D. A., N. Lehman, S. J. O'Brien and R. K. Wayne 1990. Genetic fingerprinting reflects population differentiation in the California Channel Island fox. Nature, 344: 746767.

Higuchi, A. 1976. Ryudo. In, Marine Ecology and Fishery Field in the Seto Inland Sea, Murakami, A. (chief ed.), Fuji Technosystem, pp. 41-54 (in Japanese)

Itami, M. 1984. 1. Seto Inland Sea. In, General Study for Environmental Assessment on the Seto Inland Sea. Reports of 
Co-operative Research (Preservation of Environment) Ministry of Education, Science and Culture, pp. 15-47 (in Japanese)

Miyanohara, M., Iwatsuki, Y. and Sakai, M. 1999. Analysis of the Okinawa and Miyazaki populations of the common silver-biddy Gerres oyena using random amplified polymorphic DNA (RAPD) techniques. Fisheries Science, 65: $177-181$.

Nei, M. 1975. Molecular Population Genetics and Evolution. North-Holland, Amsterdam. 288pp.

Nishikawa, T. 1995. 25-B. Cephalochordata. In, Guide to seashore animals of Japan with color pictures and keys. Vol. II. Nishimura, S. (ed.), Hoikusha, Tokyo, pp. 608-610 (in Japanese).

Nishikawa, T. 1998. Namekujiuwo. In, Databook for Rarefied Wild Aquatic Organisms in Japan, Bureau of Fisheries (ed.), Nihon Suisan Shigen Hogokyoukai, Tokyo, pp. 62-63 (in Japanese).
Shojima, Y. 1974. Gleanings of spawning research (3) lancelets (Sanran Chosa Koborebanashi (3) Namekujiuo). Seikaiku Suisan Kenkyusho News, 18: 4-7. (in Japanese)

Stokes, M. D. and N. D. Holland 1998. The lancelet. American Scientist, 86: 552-560.

Wada, H. and N. Satoh 1994. Details of the evolutionary history from invertebrates to vertebrates, as deduced from the sequence of $18 \mathrm{~S}$ rDNA. Proceedings of the National Academy of Sciences of the United States of America, 91: 18011804.

Wickstead, J. H. 1975. Chordata: Acrania (Cephalochordata). In, Reproduction of Marine Invertebrates, 2, Giese, A. C. and Pearse, J. S. (eds.), Academic Press, New York, pp. 283-319.

Yanagi, T. and A. Higuchi 1979. A. Constant current in the Seto Inland Sea. Study note on the coastal oceanography, 16: 123-127 (in Japanese). 